\title{
The Aging Midfacial Skeleton: Implications for Rejuvenation and Reconstruction Using Implants
}

\author{
E. Matros, M.D., ${ }^{1}$ A. Momoh, M.D., ${ }^{1,2}$ and M.J. Yaremchuk, M.D. ${ }^{1}$
}

ABSTRACT

Traditional theories on facial aging and methods for rejuvenation focus primarily on soft tissues with ptosis as the major mechanism responsible for senescent changes. Anatomic studies demonstrate that there are also many changes to the craniofacial skeleton as patients age. Midface skeletal augmentation, using implants made of porous polyethylene, is a simple and effective method to reverse age-related changes of the facial skeleton in patients with intact occlusion. Skeletal implants correct concave morphology by increasing projection and provide a means to resuspend cheek soft tissues that have descended off a deficient bony platform. Beyond rejuvenation, skeletal implants can be used to restore facial proportions in patients with midface deficiency secondary to trauma, congenital deformity, or other pathologic states.

KEYWORDS: Implants, aging, midface, skeleton

Traditional concepts of midface aging focus on soft tissue ptosis, secondary to gravitational forces, as the major mechanism responsible for senescent changes. Although clinically apparent long before, Yousif et al objectively documented using photogrammetry that with advancing age there is anterior and inferior descent of the cheek mass with deepening of the nasolabial fold. ${ }^{1}$ This data along with empiric observations on facial aging provides a rationale for current face-lifting techniques that remove excess skin and resuspend deep soft tissues. ${ }^{2,3}$ Recent studies suggest that ptosis explains only a portion of the soft tissue changes associated with facial aging. Lambros' longitudinal photographic analysis of 103 patients spanning an average period of 25 years demonstrates that the lid-cheek junction, brow, and soft tissue landmarks such as nevi remain fixed in position in

${ }^{1}$ Division of Plastic Surgery, Department of Surgery, Massachusetts General Hospital and Harvard Medical School, Boston, Massachusetts; ${ }^{2}$ Division of Plastic Surgery, Baylor College of Medicine, Houston, Texas.

Address for correspondence and reprint requests: Michael J. Yaremchuk, M.D., Division of Plastic Surgery, Massachusetts General Hospital, Wang 435, 15 Parkman Street, Boston, MA 02114 (e-mail: a significant proportion of patients with advancing age. ${ }^{4}$ Lambros and others conclude that changes in volume of cheek fat, either loss or gain, rather than ptosis account for an aged appearance. As such, their techniques for rejuvenation include injections with either autologous fat or allogenic fillers. ${ }^{5,6}$

The aforementioned studies address soft tissue changes only; however, a comprehensive analysis of facial aging reveals that the craniofacial skeleton undergoes many changes as individuals mature. Early studies of facial bone growth suggested the craniofacial skeleton grew until skeletal maturity, but data from more recent studies do not support this theory. ${ }^{7,8}$ Israel demonstrated in a longitudinal radiogrammetric study that the skull, cranial base, and upper facial compartment increased from adulthood into senescence of the order 5 to $7 \% .{ }^{9}$

myaremchuk@partners.org).

Management of the Aging Face; Guest Editor, Anthony P. Sclafani, M.D., F.A.C.S.

Facial Plast Surg 2009;25:252-259. Copyright (C) 2009 by Thieme Medical Publishers, Inc., 333 Seventh Avenue, New York, NY 10001, USA. Tel: +1(212) 584-4662.

DOI 10.1055/s-0029-1242037. ISSN 0736-6825. 

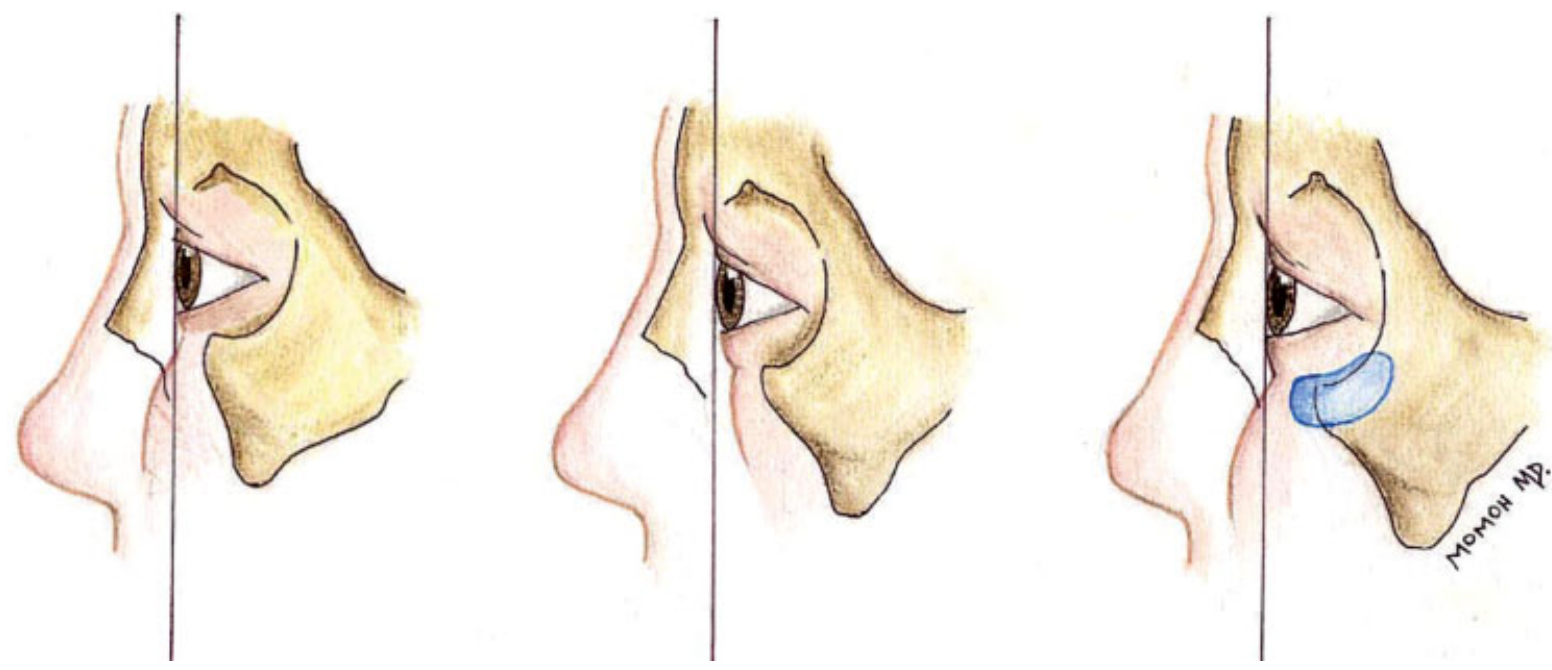

Figure 1 Anatomic variations of the anterior cheek mass and infraorbital rim are depicted. (Left) Positive vector proportions are present when the anterior cheek mass and infraorbital rim lie anterior to the corneal surface. (Middle) With advancing age or in various pathologic states, there is an increasing incidence of the negative vector deformity. ${ }^{14}$ The anterior cheek mass and infraorbital rim lie posterior to the surface of the cornea. Patients with this anatomic configuration have increased complications after lower eyelid surgery. (Right) Infraorbital rim implants correct negative vector proportions by augmenting the infraorbital rim and providing a platform to support the cheek mass.

Behrents' thesis on longitudinal craniofacial skeletal morphology concluded that growth rates decelerated but continued into the oldest age spans. ${ }^{10}$ Bartlett et al in a cross-sectional cohort analysis of 160 skulls from the Smithsonian Institution observed that facial width, depth, and height either increased or remained unchanged in older cohorts. ${ }^{11}$ These studies do not address specific anatomic facial regions but taken together support that skull growth continues, albeit at varying rates, from infancy through late adulthood.

The clinical implications of skeletofacial aging are revealed in studies of relevant anatomic areas. Using a cross-sectional cohort design, Pessa and Shaw independently observed retrusion of the lower maxilla in patients from older compared with younger cohorts. ${ }^{12,13}$ Pessa suggests that maxillary retrusion is a principal factor in development of a prominent nasolabial fold in cases of maxillary hypoplasia, Crouzon syndrome, or as part of normal facial aging. In a separate study of periorbital aging, Pessa measured that the orbital rim and anterior cheek mass lie posterior to the corneal surface in older compared with younger patients (Fig. 1). ${ }^{14}$ Citing the work of Jelks, Pessa concludes that the increased incidence of negative vector proportions in older patients contributes to complications after periorbital surgery. ${ }^{15}$ Other anatomic studies demonstrate an enlarged orbital aperture in older individuals due to curve distortion of the superomedial and inferolateral orbital rim. ${ }^{16,17}$ The authors postulate that orbital remodeling has aesthetic implications by leading to formation of crow's-feet and lower lid lag. A summary of age-related changes to the midfacial skeleton based on data from available studies is depicted in Fig. 2.
Although further research needs to be performed, age-related changes to both the overlying soft tissues and craniofacial skeleton have implications for reconstructive and aesthetic surgeons who attempt to effectively restore and rejuvenate facial appearance. Prior to undertaking surgical procedures on the face, surgeons must assess for changes at both lamella with intervention directed at the appropriate level. The current report describes the senior author's technique and indications for infraorbital rim, paranasal, and malar implant augmentation of the midface skeleton. It provides a rationale for hard tissue replacement in both facial rejuvenation and correction of a dysesthetic appearance in a subset of patients with acquired and congenital deformities.

\section{SURGICAL TECHNIQUE}

\section{Anesthesia}

General anesthesia is preferred. This approach allows adequate airway protection, surgical access, and intraoral cleansing.

\section{Access}

Infraorbital rim implants are placed through an intraoral upper sulcus and lower eyelid incisions. Both incisions are required for degloving of the midface soft tissues and infraorbital nerve visualization. The retroseptal transconjunctival incision is preferred to a preseptal approach to avoid delamination of the lower lid and scarring along the septal plane. The lateral canthotomy extension of the transconjunctival incision is avoided because it causes 

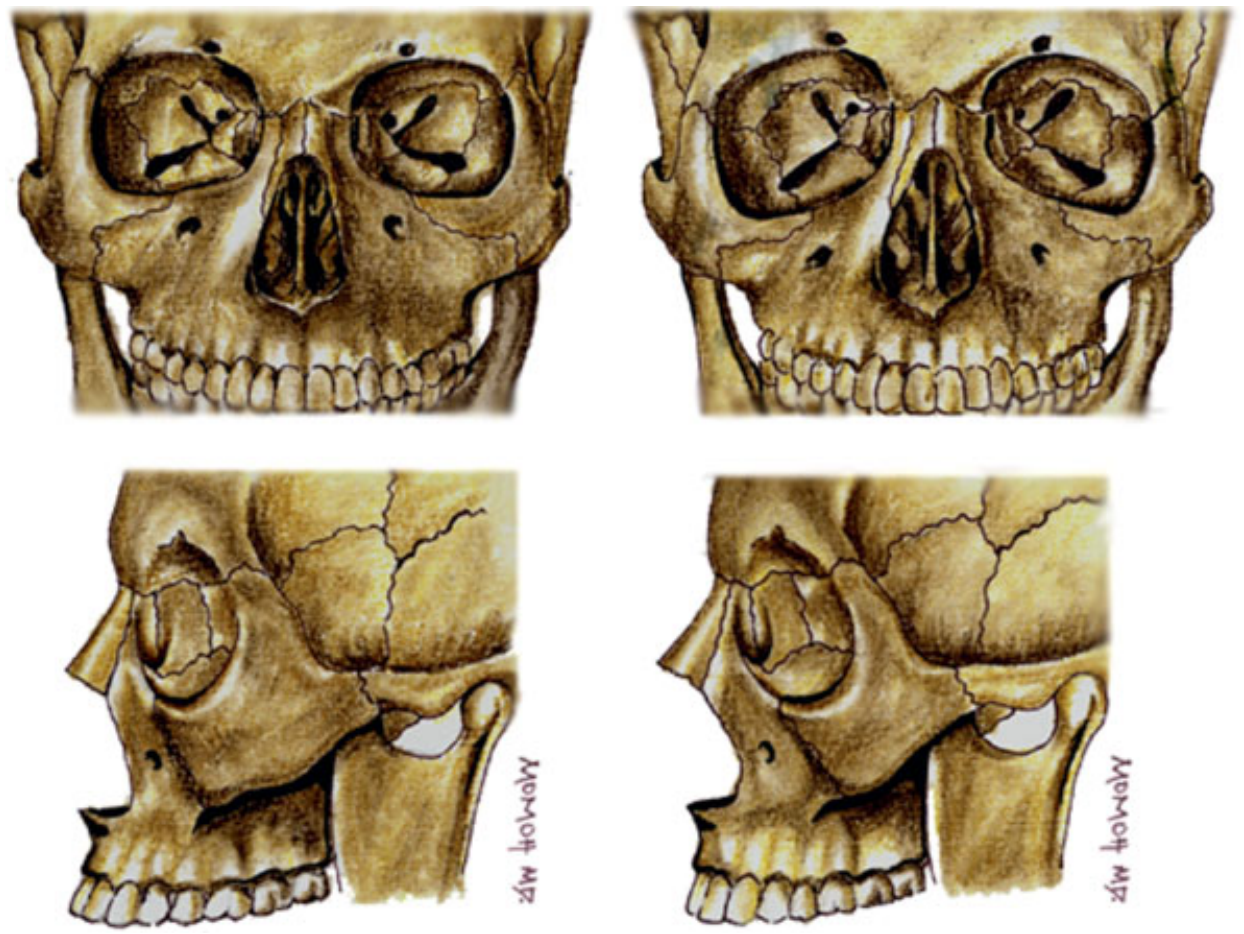

Figure 2 A pictorial summary of age-related changes to the midface skeleton is shown. (Left) Features of a youthful skull include a malar eminence, infraorbital rim, and piriform aperture that are positioned anterior and vertical in the sagittal plane. The orbital aperture is small with a horizontally positioned inferior orbital rim. (Right) Older patients have a retroclined malar eminence, infraorbital rim, and piriform aperture compared with that of young patients. The orbital aperture area is increased secondary to progressive curve distortion of orbital rim superomedially and inferolaterally. It should be noted that the implants presented herein match the skeletally deficient areas of older patients.

rounding of the lateral canthus and contributes to prolonged palpebral edema. In patients requiring a lateral canthopexy, a lateral extent of a blepharoplasty incision is added in a crow's-feet rhytide to access the zygomaticofrontal suture for a bridge of bone canthopexy (see later).

When indicated, malar and paranasal implants can be placed at the same time as infraorbital implants or used independently. Malar augmentation is performed through intraoral and lateral extent of blepharoplasty incisions. The blepharoplasty incision permits screw fixation of the implant over the proximal zygomatic arch. Placement of paranasal implants requires an intraoral incision.

\section{Soft Tissue Mobilization}

Through the access incisions, the midface soft tissues are widely undermined in the subperiosteal plane along the maxilla, extending laterally to the proximal zygomatic arch. This permits en bloc vertical resuspension of the soft tissues and lower lid while creating adequate space for an infraorbital implant.

In patients requiring a canthopexy, an additional temporal incision may be necessary for redistribution of temporal soft tissues. Performed beneath the superficial layer of the deep temporal fascia, this dissection proceeds until the zygomatic arch and lateral orbital rim are reached.

\section{Implant Placement}

The infraorbital implant (Porex Surgical, Newman, GA) is the most important implant to correct midface deformities, achieving 3 to $5 \mathrm{~mm}$ of anterior projection (Fig. 3). The implant flange allows stabilization on the anterior edge of the orbital floor and provides an area for screw fixation. Up to three implants, including paranasal and malar, may be necessary depending on the aesthetic needs of the patient. To closely follow the complex contour of the midface and avoid infraorbital nerve impingement, use of multiple implants is preferable to use of a single large implant. Specific paranasal implants placed along the piriform aperture have been designed (Fig. 4) ${ }^{18}$ If the area lateral or inferior to the infraorbital implant is hollow, a malar implant is fashioned (Fig. 5). Implants are fixed to the skeleton with titanium screws and contoured in situ with a burr for seamless transition with the skeleton and adjacent implants.

\section{Lateral Canthopexy}

Lateral canthopexy is indicated for patients with lid malposition and shape distortion of the lateral canthus 

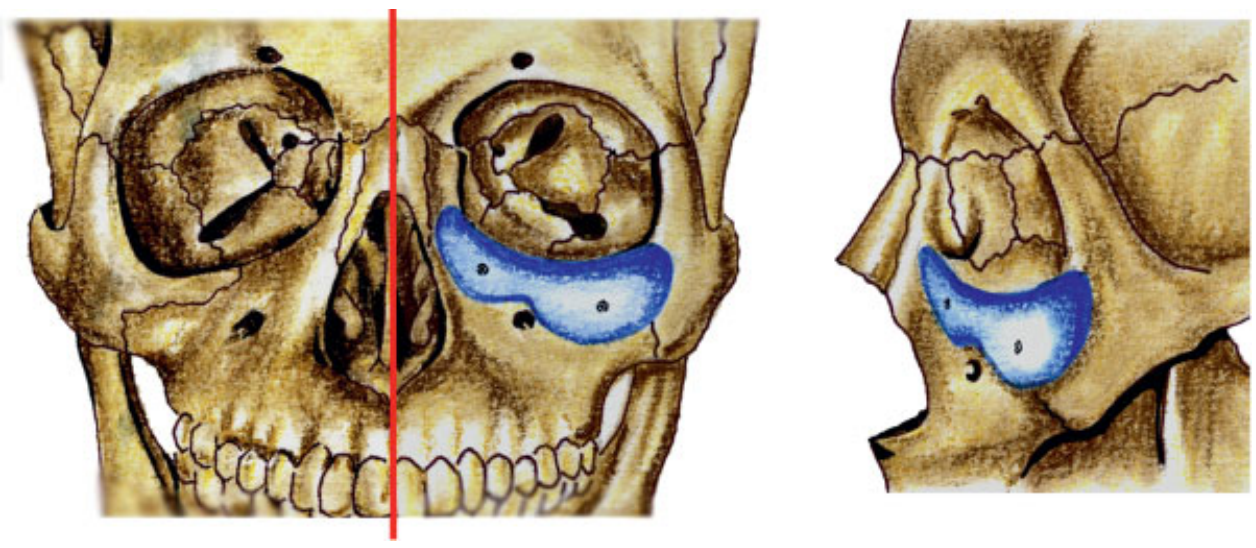

Figure 3 Infraorbital implants are the most important implants used in midface skeletal augmentation. Placed through combined intraoral and transconjunctival incisions, these implants increase infraorbital projection in patients with negative vector proportions. Concomitant subperiosteal degloving allows for resuspension of the cheek soft tissues via suturing to the implant. Infraorbital implants are indicated as part of facial rejuvenation or improvement of midface proportions in trauma, congenital deformity, or skeletal variation.
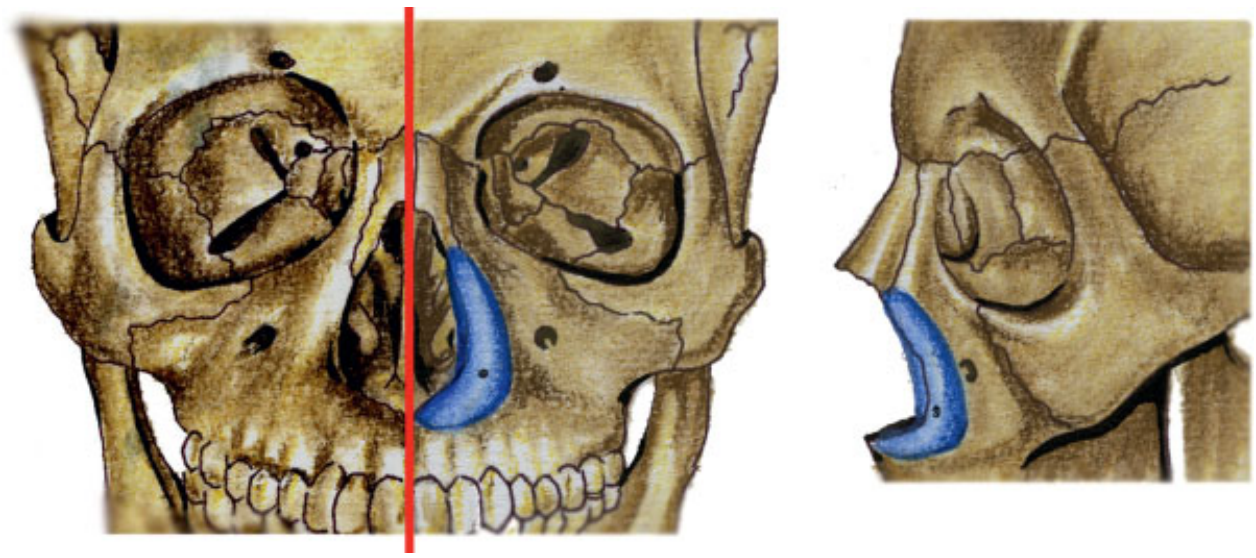

Figure 4 Paranasal implants can be used in patients who benefit from Lefort I advancement but have intact occlusion. This implant is placed along the piriform aperture via an intraoral upper sulcus incision. Implants in this location can be used in patients with Binder syndrome, in ethnic groups, in those with congenital conditions, or after posttraumatic deformity.
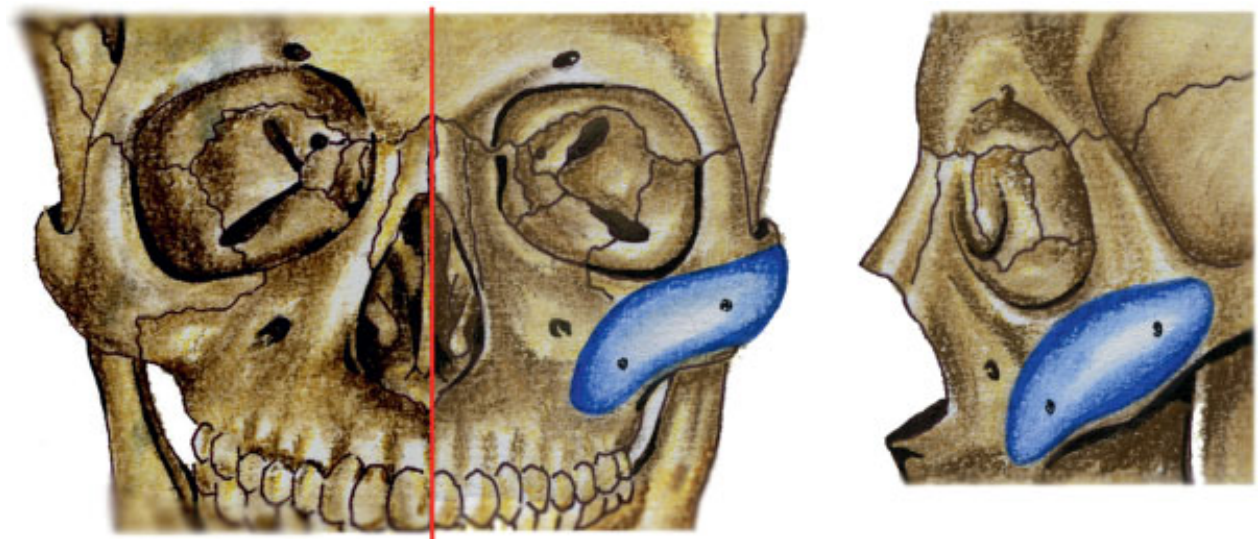

Figure 5 Malar implants can be used in isolation or in combination with other skeletal implants. These implants are placed through intraoral and lateral extent of blepharoplasty incisions to increase projection in the region lateral or inferior to an infraorbital implant. Malar implants are frequently used for aesthetic purposes and congenital or acquired midface deformities. 

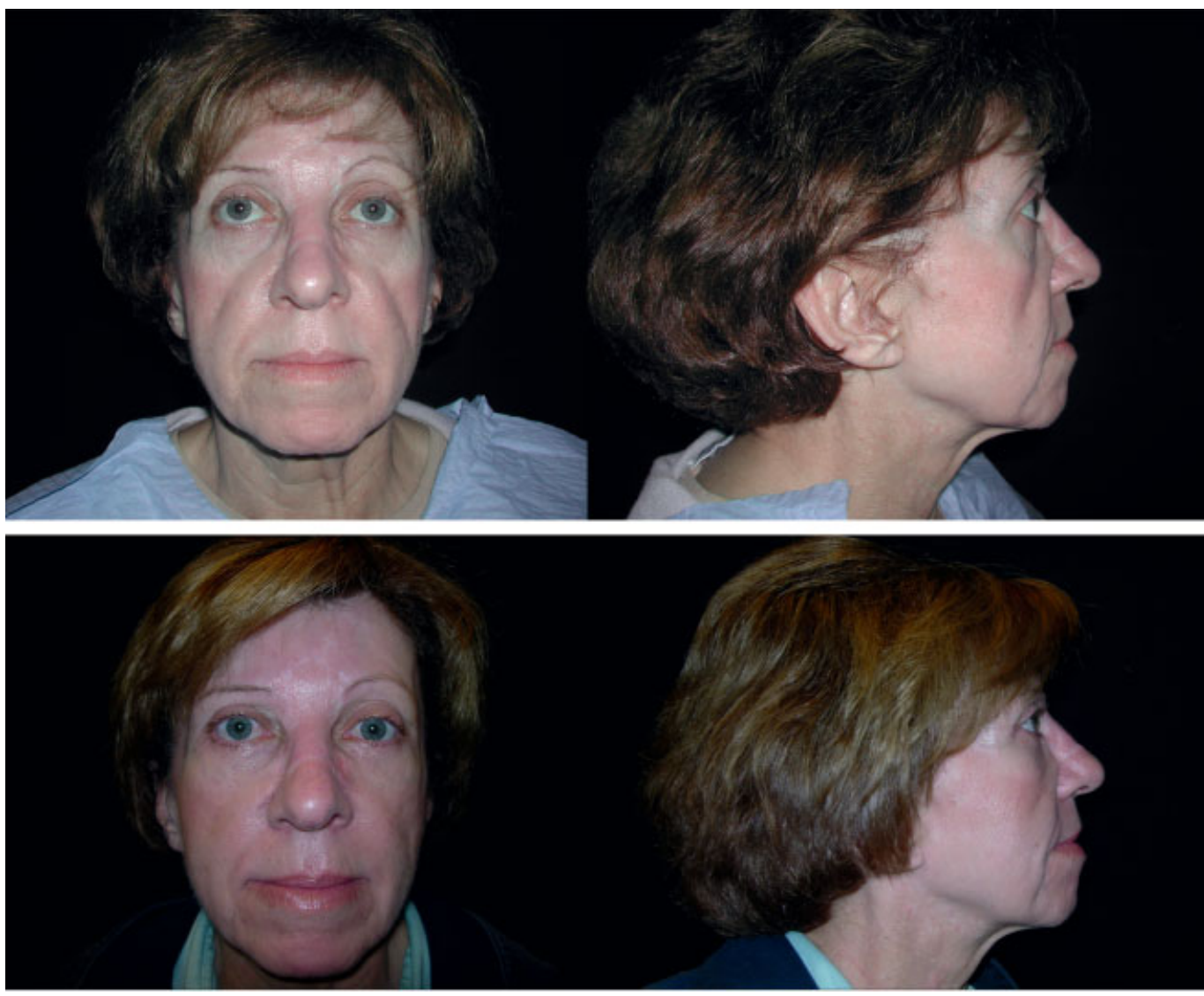

Figure 6 A 63-year-old woman with negative vector proportions and ptosis of cheek soft tissues underwent infraorbital rim augmentation with midface resuspension. (Top) Preoperative frontal and lateral views. (Bottom) Postoperative views.

after blepharoplasty or with a congenitally low-lying lateral canthus. Through the lateral extent of the lower blepharoplasty incision, the zygomatic frontal suture is exposed with drill holes placed $2 \mathrm{~mm}$ above and at the suture itself. A figure-of-eight, 30-gauge titanium wire suture is then passed through the lateral canthus with each end placed through the holes in the lateral orbital wall. The ends of the wire are twisted around the bridge of bone and secured behind the lateral orbital rim. ${ }^{19}$ The final lateral canthus position should lie 2 to $3 \mathrm{~mm}$ above the medial canthal plane.

\section{Soft Tissue Elevation}

Two sutures are used to elevate the cheek and lower lid soft tissues. Sutures are placed in the periosteum adherent to the midface soft tissue, $3 \mathrm{~cm}$ below both the midpupillary line and lateral canthus. The midpupil suture is secured to the infraorbital rim implant, whereas the lateral suture is passed through either the lateral aspect of the infraorbital implant or a drill hole in the lateral orbital rim.

For patients who undergo lateral canthopexy, the temporal soft tissues are undermined and redistributed to avoid bunching that may occur in the region along the lateral upper lid or brow margin. Remaining excess tissue can be excised through either a lateral brow lift or upper lid blepharoplasty incisions.

Closed suction drains are placed beneath the soft tissues and brought out through a stab incision in the temporal scalp or retroauricular area. A temporary tarsorrhaphy is placed to minimize chemosis. Postoperative antibiotics are continued for 5 days.

\section{Clinical Examples}

Preoperative and postoperative photographs of patients who have undergone skeletal augmentation with porous polyethylene midfacial implants are shown in Figs. 6 and 7.

\section{DISCUSSION}

Midface concavity may be the clinical end point of either pathologic or nonpathologic craniofacial processes. It may reflect a deformity secondary to an underlying craniosynostosis syndrome such as Apert or Crouzon syndrome, trauma, extreme forms of skeletal variation, or be part of normal facial aging. Whereas Lefort I and III osteotomies with advancement are used to restore facial proportions and occlusion in patients with craniofacial dysostoses, other etiologies of midface concavity can be 

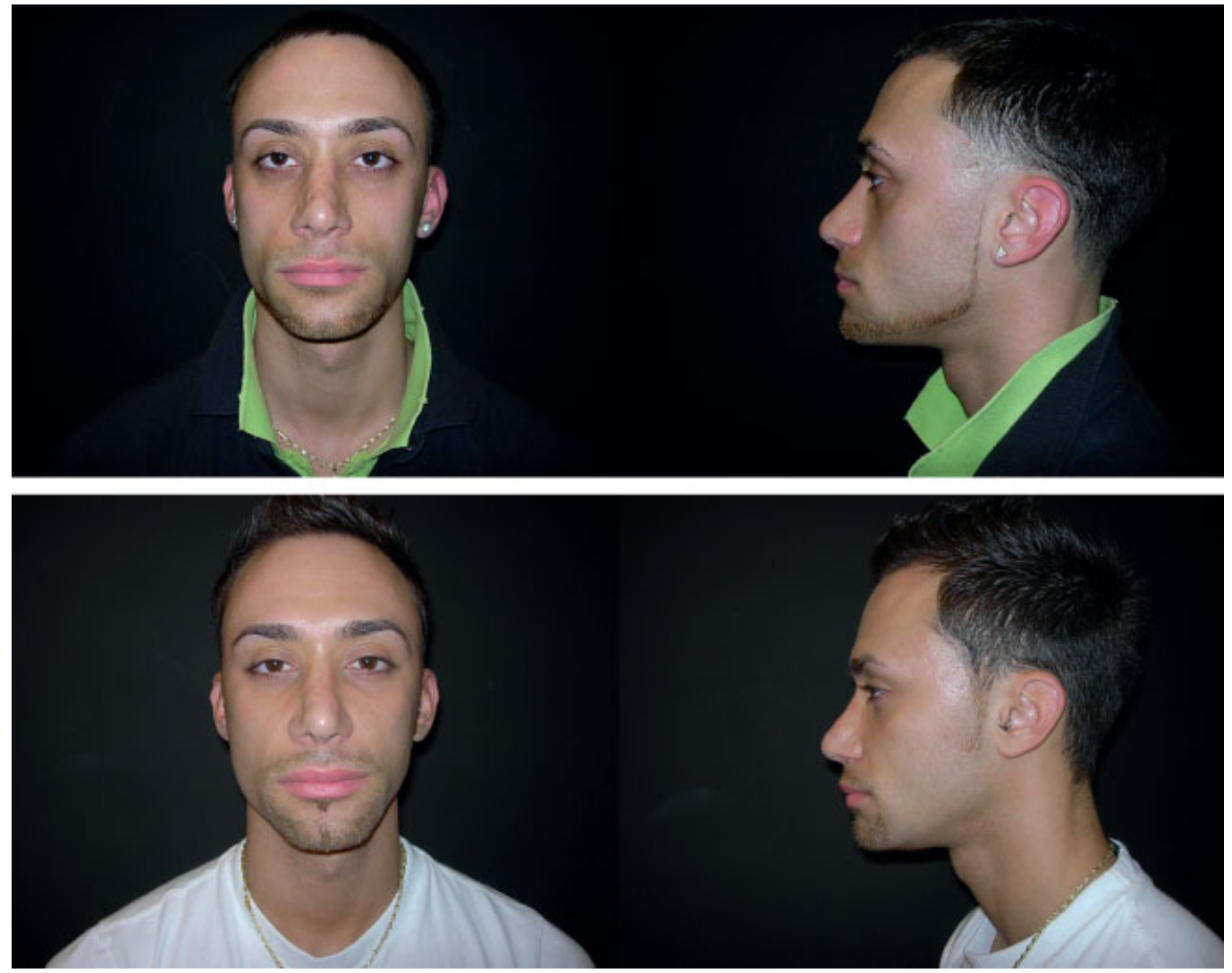

Figure 7 A 28-year-old man with midface hypoplasia, tired appearance, and premature signs of aging underwent surgical correction with combined use of infraorbital rim, malar, and paranasal implants, and midface resuspension. (Top) Preoperative frontal and lateral views. (Bottom) Postoperative views.

treated with skeletal augmentation as the occlusion is intact.

Patients with midface concavity have premature and exaggerated signs of aging. ${ }^{14,19-22}$ They appear tired due to the presence of long lower eyelids, loss of cheek prominence, rounding of the lateral canthal angle, and increased vertical height of the palpebral fissure. The mechanism whereby midface skeletal deficiency contributes to these overlying soft tissue changes is unclear, but it may be that decreased bony projection hastens the gravitational descent of an unsupported soft tissue envelope. ${ }^{14}$ An alternate hypothesis is proposed by Levine et al who contend that soft tissue descent is the "engine" driving the aging process with bone relocation and reshaping determined by the pull of surrounding soft tissues. $^{23}$ Regardless of the pathophysiologic mechanism, skeletal augmentation provides a means to effectively restore facial convexity and reestablish a platform whereby facial soft tissues can be supported.

Skeletal facial implants facilitate conversion of concave into convex faces in two ways. By augmenting skeletally deficient areas, projection is increased. Depending upon the indication, multiple implants may be needed to restore facial proportions. Second, subperiosteal midface resuspension, performed at time of infraorbital augmentation, has both direct and indirect effects.
Direct elevation of tissues through suspension sutures restores cheek fullness, which is abnormally positioned in aging or various pathologic states. Indirect changes are notable in the periorbital region where restoration of cheek bulk secondarily elevates the cheek-lid junction. This restores features of a youthful periorbita such as a short lower eyelid, narrow palpebral fissure, and with the use of a lateral canthopexy an acute lateral canthal angle. ${ }^{19,24,25}$

Although clinicians commonly assess the face in the coronal plane, indications for skeletal augmentation, particularly infraorbital implants, are best understood by examination in the sagittal view. Patients with midface deficiency, regardless of etiology, have an altered sagittal globe-orbital rim relationship. ${ }^{14,26}$ The clinical relevance of the sagittal globe-orbital rim relationship was recognized indirectly by Rees and LaTrenta who measured an association between patients with prominent eyes, scleral show, maxillary hypoplasia or lower lid hypotonia and dry eye symptoms after blepharoplasty. ${ }^{27}$ These patients were considered "morphologically prone" to dry eye symptoms and required special operative considerations. Jelks and Jelks in 1993 more formally defined the sagittal globeorbital rim relationship and suggested unfavorable results after blepharoplasty in patients with "negative 
vector" proportions. ${ }^{15}$ The authors define "negative vector" morphology as a malar eminence that lies posterior to the globe surface, whereas a "positive vector" is when the malar eminence lies anterior to the globe surface. More recently, Pessa and others have shown that aging contributes to the negative vector deformity through posterior positioning of the inferior orbital rim and anterior maxillary wall (Fig. 2). ${ }^{12-14,28}$ Figures 1 and 6 demonstrate correction of the negative vector deformity using infraorbital rim augmentation. Infraorbital rim implants are indicated as part of facial rejuvenation or for correction of midface deficiency, prominent eyes, and palpebral distortion after lower blepharoplasty. ${ }^{19-21,29}$

Paranasal and malar implants are recommended for patients with lower midface concavity who would benefit from Lefort I advancement but have intact occlusion. Although paranasal and malar implants are commonly combined with infraorbital rim implants (Fig. 7), either can be used in isolation. ${ }^{18}$ Paranasal augmentation with or without rhinoplasty is described for patients with nasomaxillary deficiency such as Binder syndrome. ${ }^{30,31}$ Similarly, patients with cleft lip may have an altered lip and nasal relationship from prior surgery or abnormal maxillary growth, which can be corrected by paranasal implants. Asians and African Americans are a subset of ethnic patients with flat facial profiles or large noses, which can be effectively camouflaged with implants placed along the piriform aperture. Whitaker reported on 106 patients who underwent malar augmentation alone for midface hypoplasia. ${ }^{32}$ Implants were placed from the region lateral to the piriform to the zygomatic arch. Indications included aesthetic improvement in greater than $50 \%$ and reconstruction for either hemifacial microsomia or posttraumatic deformity in most other patients.

Midface projection can be restored through use of either bone grafts or alloplastic materials. Bone grafts are unpredictable because the entirety of graft may not revascularize or once incorporated becomes subject to osteoclastic activity that is under the influence of deforming forces (Wolffs law). ${ }^{33}$ In addition, bone grafts have associated donor-site morbidity such as pain and contour irregularities as well as the increased cost of operative time necessary for harvesting. Alloplastic materials are preferable because they are stable and lack donor-site morbidity.

Various alloplastic materials can be used for facial augmentation. Unlike smooth implants, which generate capsules that can irreversibly distort soft tissues, porous polyethylene due to its pore size of 100 to $250 \mu \mathrm{m}$ tends to have improved tissue in-growth and revascularization through its pores. ${ }^{34}$ The low infection and extrusion rates associated with porous polyethylene may be due to soft tissue in-growth. ${ }^{35}$ Porous polyethylene as a biomaterial has good handling properties such that once fixed in position, it can be carved and contoured in situ using a burr.

Reliable and reproducible technical results with facial augmentation can be achieved by screw fixation using multiple implants. Use of more than one implant allows precise adaptation to the curvature of the face without gaps, which lead to unanticipated excess augmentation. Multiple implants also avoids impingement on the infraorbital nerve. Screw fixation prevents secondary movement of the implant and aids in situ contouring at junctions with the skeleton and other implants.

In conclusion, skeletal augmentation is a useful tool for surgeons to either restore or rejuvenate facial appearance in patients with congenital, acquired, or aesthetic deformities. Use of alloplastic material is straightforward and affords the surgeon an opportunity to resuspend soft tissues that have descended on a hypoplastic skeletal platform. Although the indications for augmentation in pathologic states may not change significantly in the years to come, as more is learned about skeletal aging in larger longitudinal studies, there may be an expanding role for skeletal augmentation in facial rejuvenation.

\section{DISCLOSURE}

Dr. Yaremchuk receives royalties from Porex Surgical, a company that manufactures facial implants. The other authors have no financial interests in this research project or in any of the techniques or equipment used in this study. The authors have no conflicts of interest to disclose.

\section{REFERENCES}

1. Yousif NJ, Gosain A, Sanger JR, et al. The nasolabial fold: a photogrammetric analysis. Plast Reconstr Surg 1994;93: 70-77

2. Hamra ST. The deep-plane rhytidectomy. Plast Reconstr Surg 1990;86:53-61; discussion 62-63

3. Baker DC. Lateral SMASectomy. Plast Reconstr Surg 1997;100:509-513

4. Lambros V. Observations on periorbital and midface aging. Plast Reconstr Surg 2007;120:1367-1376; discussion 1377

5. Coleman SR. Facial recontouring with lipostructure. Clin Plast Surg 1997;24:347-367

6. Lambros VS. Hyaluronic acid injections for correction of the tear trough deformity. Plast Reconstr Surg 2007;120(6 Suppl): $74 \mathrm{~S}-80 \mathrm{~S}$

7. Tallgren A. Neurocranial morphology and ageing-a longitudinal Roentgen cephalometric study of adult Finnish women. Am J Phys Anthropol 1974;41:285-293

8. Goldstein M. Changes in dimensions and form of the face and head with age. Am J Phys Anthropol 1936;22: 37-89

9. Israel H. Recent knowledge concerning craniofacial aging. Angle Orthod 1973;43:176-184 
10. Behrents RG. Growth in the Aging Craniofacial Skeleton. Ann Arbor, MI: Center for Human Growth and Development, The University of Michigan; 1985

11. Bartlett SP, Grossman R, Whitaker LA. Age-related changes of the craniofacial skeleton: an anthropometric and histologic analysis. Plast Reconstr Surg 1992;90:592-600

12. Pessa JE, Zadoo VP, Mutimer KL, et al. Relative maxillary retrusion as a natural consequence of aging: combining skeletal and soft-tissue changes into an integrated model of midfacial aging. Plast Reconstr Surg 1998;102:205-212

13. Shaw RB, Jr, Kahn DM. Aging of the midface bony elements: a three-dimensional computed tomographic study. Plast Reconstr Surg 2007;119:675-681; discussion 82-83

14. Pessa JE, Desvigne LD, Lambros VS, Nimerick J, Sugunan B, Zadoo VP. Changes in ocular globe-to-orbital rim position with age: implications for aesthetic blepharoplasty of the lower eyelids. Aesthetic Plast Surg 1999;23: 337-342

15. Jelks GW, Jelks EB. Preoperative evaluation of the blepharoplasty patient. Bypassing the pitfalls. Clin Plast Surg 1993;20:213-223; discussion 224

16. Pessa JE, Chen Y. Curve analysis of the aging orbital aperture. Plast Reconstr Surg 2002;109:751-755; discussion 756-760

17. Kahn DM, Shaw RB Jr. Aging of the bony orbit: a threedimensional computed tomographic study. Aesthet Surg J 2008;28:258-264

18. Yaremchuk MJ, Israeli D. Paranasal implants for correction of midface concavity. Plast Reconstr Surg 1998;102:16761684; discussion 1685

19. Yaremchuk MJ. Restoring palpebral fissure shape after previous lower blepharoplasty. Plast Reconstr Surg 2003; 111:441-450; discussion 451-452

20. Yaremchuk MJ. Infraorbital rim augmentation. Plast Reconstr Surg 2001;107:1585-1592; discussion 1593-1595

21. Yaremchuk MJ. Improving periorbital appearance in the “morphologically prone". Plast Reconstr Surg 2004;114:980987

22. Pessa JE, Zadoo VP, Yuan C, et al. Concertina effect and facial aging: nonlinear aspects of youthfulness and skeletal remodeling, and why, perhaps, infants have jowls. Plast Reconstr Surg 1999;103:635-644
23. Levine RA, Garza JR, Wang PT, Hurst CL, Dev VR. Adult facial growth: applications to aesthetic surgery. Aesthetic Plast Surg 2003;27:265-268

24. Farkas LG, Hreczko TA, Katic MJ. Craniofacial Norms in North American Caucasians from Birth to Adulthood. Appendix A Anthropometry of the Head and Face. 2nd ed. New York, NY: Raven Press; 1994:241-333

25. Yaremchuk MJ. Subperiosteal and full-thickness skin rhytidectomy. Plast Reconstr Surg 2001;107:1045-1058

26. Mulliken JB, Godwin SL, Pracharktam N, Altobelli DE. The concept of the sagittal orbital-globe relationship in craniofacial surgery. Plast Reconstr Surg 1996;97: 700-706

27. Rees TD, LaTrenta GS. The role of the Schirmer's test and orbital morphology in predicting dry-eye syndrome after blepharoplasty. Plast Reconstr Surg 1988;82:619-625

28. Shaw RB Jr, Kahn DM. Aging of the midface bony elements: a three-dimensional computed tomographic study. Plast Reconstr Surg 2007;119:675-681; discussion 682-683

29. Yaremchuk MJ. Making concave faces convex. Aesthetic Plast Surg 2005;29:141-147; discussion 148

30. Converse JM, Horowitz SL, Valauri AJ, Montandon D. The treatment of nasomaxillary hypoplasia. A new pyramidal naso-orbital mazillary osteotomy. Plast Reconstr Surg 1970; 45:527-535

31. Jackson IT, Moos KF, Sharpe DT. Total surgical management of Binder's syndrome. Ann Plast Surg 1981;7:25-34

32. Whitaker LA. Aesthetic augmentation of the malar-midface structures. Plast Reconstr Surg 1987;80:337-346

33. Chen NT, Glowacki J, Bucky LP, Hong HZ, Kim WK, Yaremchuk MJ. The roles of revascularization and resorption on endurance of craniofacial onlay bone grafts in the rabbit. Plast Reconstr Surg 1994;93:714-722; discussion 723-724

34. Wellisz T, Kanel G, Anooshian R. Characteristics of the tissue response to Medpor porous polyethylene implants in the human facial skeleton. J Long Term Eff Med Implants 1993;3:223-235

35. Rubin JP, Yaremchuk MJ. Complications and toxicities of implantable biomaterials used in facial reconstructive and aesthetic surgery: a comprehensive review of the literature. Plast Reconstr Surg 1997;100:1336-1353 